\title{
The solar photospheric abundance of phosphorus:
} results from $\mathrm{CO}^{5} \mathrm{BOLD} 3 \mathrm{D}$ model atmospheres ${ }^{\star}$

\author{
E. Caffau ${ }^{1}$, M. Steffen ${ }^{2}$, L. Sbordone ${ }^{1,3}$, H.-G. Ludwig ${ }^{1,3}$, and P. Bonifacio ${ }^{1,3,4}$ \\ 1 GEPI, Observatoire de Paris, CNRS, Université Paris Diderot, Place Jules Janssen, 92190 Meudon, France \\ e-mail: Elisabetta.Caffau@obspm. fr \\ 2 Astrophysikalisches Institut Potsdam, An der Sternwarte 16, 14482 Potsdam, Germany \\ 3 CIFIST Marie Curie Excellence Team \\ 4 Istituto Nazionale di Astrofisica, Osservatorio Astronomico di Trieste, via Tiepolo 11, 34143 Trieste, Italy \\ Received 27 July 2007 / Accepted 11 August 2007
}

\section{ABSTRACT}

\begin{abstract}
Aims. We determine the solar abundance of phosphorus using $\mathrm{CO}^{5} \mathrm{BOLD} 3 \mathrm{D}$ hydrodynamic model atmospheres. Methods. High-resolution, high signal-to-noise solar spectra of the PI lines of Multiplet 1 at 1051-1068 nm are compared to lineformation computations performed on a $\mathrm{CO}^{5} \mathrm{BOLD}$ solar model atmosphere.

Results. We find $A(\mathrm{P})=5.46 \pm 0.04$, in good agreement with previous analyses based on 1D model atmospheres, due to the P I lines of Mult. 1 not being affected much by 3D effects. We cannot confirm an earlier claim by other authors of a downward revision of the solar $\mathrm{P}$ abundance by 0.1 dex when employing a 3D model atmosphere.

Concerning other stars, we find modest $(<0.1$ dex $) 3 \mathrm{D}$ abundance corrections for $\mathrm{P}$ among four F-dwarf model atmospheres of different metallicities, and these corrections are largest at lowest metallicity.

Conclusions. We conclude that 3D abundance corrections are generally rather small for the PI lines studied in this work. They are marginally relevant for metal-poor stars, but may be neglected in the Sun.
\end{abstract}

Key words. Sun: abundances - stars: abundances - hydrodynamics

\section{Introduction}

Phosphorus is an element whose nucleosynthesis is not clearly understood and whose abundance is very poorly known outside the solar system. It has a single stable isotope ${ }^{31} \mathrm{P}$, and its most likely sites of production are carbon and neon burning shells in the late stages of the evolution of massive stars, which end up as Type II SNe. The production mechanism probably occurs via neutron capture, as it is for the parent nuclei ${ }^{29} \mathrm{Si}$ and ${ }^{30} \mathrm{Si}$. According to Woosley \& Weaver (1995), there is no significant $\mathrm{P}$ production during the explosive phases.

Like for other odd- $\mathrm{Z}$ elements $(\mathrm{Na}, \mathrm{Al})$, one expects that the $\mathrm{P}$ abundance should be proportional to the neutron excess $\eta^{1}$. As metallicity decreases, the neutron excess decreases, and the abundance of odd-Z elements should decrease more rapidly than neighbouring even- $Z$ elements. One can therefore expect decreasing $\left[{ }^{31} \mathrm{P} /{ }^{30} \mathrm{Si}\right]$ or $\left[{ }^{31} \mathrm{P} /{ }^{12} \mathrm{Mg}\right]$ ratio with decreasing metallicity. Such behaviour is in fact observed for $\mathrm{Na}$ (Andrievsky et al. 2007); however, at low metallicity $[\mathrm{Na} / \mathrm{Mg}]$ seems to reach a plateau at about $[\mathrm{Na} / \mathrm{Mg}] \sim-0.5$. One could expect similar behaviour from $P$.

Phosphorus abundances in stars have so far been determined for object classes that are not apt for studies of chemical evolution: chemically peculiar stars (e.g. Castelli et al. 1997; Fremat \& Houziaux 1997, for a review of observations in $\mathrm{Hg}-\mathrm{Mn}$

* Tables 2-4 are only available in electronic form at http: //www . aanda.org

1 As defined in Arnett (1971): $\eta=\left(n_{\mathrm{n}}-n_{\mathrm{p}}\right) /\left(n_{\mathrm{n}}+n_{\mathrm{p}}\right)$, where $n_{\mathrm{n}}$ represents the total number of neutrons per gramme, both free and bound in nuclei, and $n_{\mathrm{p}}$ the corresponding number for protons. stars, see Takada-Hidai 1991 and references therein), horizontal branch stars (Behr et al. 1999; Bonifacio et al. 1995; Baschek \& Sargent 1976), sub-dwarf B type stars (Ohl et al. 2000; Baschek et al. 1982), Wolf-Rayet stars (Marcolino et al. 2007), PG 1159 stars (Jahn et al. 2007), and white dwarfs (Chayer et al. 2005; Dobbie et al. 2005; Vennes et al. 1996). Such stars are not useful for tracing the chemical evolution because either they are chemically peculiar or they are evolved and may have changed their original chemical composition. A few measurements exist for O super-giants (Bouret et al. 2005; Crowther et al. 2002) and B type stars (Tobin \& Kaufmann 1984). Kato et al. (1996) measured P in Procyon, and Sion et al. (1997) measured it in the dwarf nova VW Hydri.

The abundance of $\mathrm{P}$ in $\mathrm{F}, \mathrm{G}$, and $\mathrm{K}$ stars, which could be derived from the high-excitation IR PI lines of Mult. 1 at 1051-1068 nm, has never been explored, due to the few highresolution, near-IR spectrographs available. With the advent of the CRIRES spectrograph at the VLT, the situation is likely to change, making it possible to study the chemical evolution of $P$ in the Galaxy using these long-lived stars as tracers. With this in mind, it would be interesting as a reference to revisit the solar $\mathrm{P}$ abundance in the light of recent advances in 3D hydrodynamical model atmospheres.

There are several determinations of the solar $\mathrm{P}$ abundance in the literature. Given the difficulty of this observation, it is not surprising that $\mathrm{P}$ was absent from the seminal work of Russell (1929). Lambert \& Warner (1968) measured an abundance $A(\mathrm{P})^{2}=5.43$, using 13 lines of $\mathrm{P} \mathrm{I}$, of which four belong to

\footnotetext{
${ }^{2} A(\mathrm{P})=\log \left(N_{\mathrm{P}} / N_{\mathrm{H}}\right)+12$.
} 
Table 1. Infra-red phosphorus lines considered in this work.

\begin{tabular}{rlrr}
\hline \hline $\begin{array}{r}\text { Wavelength } \\
{[\mathrm{nm}]}\end{array}$ & Transition & $\begin{array}{r}E_{\text {low }} \\
{[\mathrm{eV}]}\end{array}$ & $\log g f$ \\
\hline 1051.1584 & $4 \mathrm{~s}^{4} \mathrm{P}_{1 / 2}-4 \mathrm{p}^{4} \mathrm{D}_{3 / 2}^{0}$ & 6.94 & -0.13 \\
1052.9522 & $4 \mathrm{~s}^{4} \mathrm{P}_{3 / 2}-4 \mathrm{p}^{4} \mathrm{D}_{5 / 2}^{0}$ & 6.95 & 0.24 \\
1058.1569 & $4 \mathrm{~s}^{4} \mathrm{P}_{5 / 2}-4 \mathrm{p}^{4} \mathrm{D}_{7 / 2}^{0}$ & 6.99 & 0.45 \\
1059.6900 & $4 \mathrm{~s}^{4} \mathrm{P}_{1 / 2}-4 \mathrm{p}^{4} \mathrm{D}_{1 / 2}^{0}$ & 6.94 & -0.21 \\
1068.1402 & $4 \mathrm{~s}^{4} \mathrm{P}_{3 / 2}-4 \mathrm{p}^{4} \mathrm{D}_{3 / 2}^{0}$ & 6.95 & -0.19 \\
\hline
\end{tabular}

Mult. 1. The oscillator strengths they used for these lines are very close to the Biemont \& Grevesse (1973) values (see Table 3). Lambert \& Luck (1978) measured $A(\mathrm{P})=5.45 \pm 0.03$, which is essentially the photospheric abundance adopted in the authoritative Anders \& Grevesse (1989) compilation $(A(\mathrm{P})=5.45 \pm 0.04)$. Based on their ab initio computed oscillator strengths Biemont et al. (1994) derived $A(\mathrm{P})=5.45 \pm 0.06$. With computed oscillator strengths, corrected to match measured energy-level lifetimes, Berzinsh et al. (1997) derived 5.49 \pm 0.04 . In their compilation, Grevesse \& Sauval (1998) adopt $A(\mathrm{P})=5.45 \pm 0.04$. As can be seen, all determinations of the solar photospheric phosphorus abundance agree within the stated uncertainties. In disagreement with earlier work, however, Asplund et al. (2005) obtained a significantly lower value of $A(\mathrm{P})=5.36 \pm 0.04$ using a 3D solar model atmosphere computed with the Stein \& Nordlund code (see Nordlund \& Stein 1997). This result might immediately be interpreted as indicating that 3D abundance corrections lead to a lower solar phosphorus abundance by $\sim 0.1 \mathrm{dex}$, but other factors, such as the choice of $\log g f$ values, can also explain this low $A(\mathrm{P})$. The $A(\mathrm{P})$ determinations found in the literature are summarised in Table 2.

In the present paper we use a solar $3 \mathrm{D} \mathrm{CO}^{5} \mathrm{BOLD}$ model to re-assess the solar phosphorus abundance. For comparison we also consider 1D solar models. Besides the Sun, we then go on to discuss the abundance corrections obtained for a number of F-dwarf models of varying metallicity.

\section{Atomic data}

We consider five IR PI lines of Mult. 1 (see Table 1). Several determinations of the oscillator strengths for this multiplet are available in the literature (see Table 3). Generally, all values are rather close, with differences smaller than 0.1 dex for any line except the $1051.2 \mathrm{~nm}$ line, for which the maximum difference is 0.18 dex. In this work we have adopted the values computed by Berzinsh et al. (1997), corrected semi-empirically, and they are, in our opinion, the best currently available. Had we chosen another set of values, with the notable exception of the Berzinsh et al. (1997) ab initio values, the difference in the derived phosphorus abundance would be 0.05 dex at most.

\section{Models}

Our analysis is based on a 3D model atmosphere computed with the $\mathrm{CO}^{5} \mathrm{BOLD}$ code (Freytag et al. 2002; Wedemeyer et al. 2004). In addition to the $\mathrm{CO}^{5} \mathrm{BOLD}$ hydrodynamical simulation, we used several 1D models. More details about these models can be found in Caffau et al. (2007) and Caffau \& Ludwig (2007). In the following we refer to the $1 \mathrm{D}$ model derived by a horizontal and temporal averaging of the $3 \mathrm{D} \mathrm{CO} \mathrm{CO}^{5} \mathrm{BOLD}$ model as the $\langle 3 \mathrm{D}\rangle$ model. The reference $1 \mathrm{D}$ model for computing the $3 \mathrm{D}$ abundance corrections is a related (same $T_{\text {eff }}, \log g$, and chemical composition) hydrostatic 1D model atmosphere computed with the LHD code, hereafter denoted as $1 \mathrm{D}_{\text {LHD }}$ model. LHD is a Lagrangian 1D hydrodynamical model atmosphere code that employs the same micro-physics as $\mathrm{CO}^{5} \mathrm{BOLD}$. Convection is treated within the mixing-length approach.

In the LHD model computation, as in any $1 \mathrm{D}$ theoretical atmosphere model, at least one free parameter describing the treatment of convection must be decided upon: we used a mixinglength parameter of $\alpha=1.25$ in the solar case, a value of $\alpha=1.8$ in $[\mathrm{M} / \mathrm{H}]=-2.0$, and $\alpha=1.0$ in $[\mathrm{M} / \mathrm{H}]=-3.0$ models. We employed the formulation of Mihalas (1978) for the mixing-length theory and neglected turbulent pressure in the momentum equation. We also considered the solar ATLAS 9 model computed by Fiorella Castelli ${ }^{3}$ and the empirical Holweger-Müller solar model (Holweger 1967; Holweger \& Müller 1974).

The spectrum synthesis codes employed are Linfor $3 \mathrm{D}^{4}$ for all models and SYNTHE (Kurucz 1993b, 2005a) in its Linux version (Sbordone et al. 2004; Sbordone 2005) for the fitting procedure with $1 \mathrm{D}$ or $\langle 3 \mathrm{D}\rangle$ models. The advantage of using SYNTHE over the present version of Linfor3D is that it can easily handle a large number of lines, thus allowing numerous weak blends to be taken into account.

The 3D $\mathrm{CO}^{5} \mathrm{BOLD}$ solar model we used is the same as described in Caffau \& Ludwig (2007). It covers a time interval of $6000 \mathrm{~s}$, represented by 25 snapshots. This covers about 10 convective turn-over time scales and 20 periods of the 5 min oscillations, which are present in the 3D model as acoustic modes of the computational box.

As in our study of sulphur (see Caffau \& Ludwig 2007), we define as "3D abundance correction" the difference in the abundance derived from the full 3D model and the related $1 \mathrm{D}_{\mathrm{LHD}}$ model, in the sense $A(3 \mathrm{D})-A\left(1 \mathrm{D}_{\mathrm{LHD}}\right)$, both synthesised with Linfor3D. We also consider the difference in the abundance derived from the $3 \mathrm{D}$ model and the $\langle 3 \mathrm{D}\rangle$ model. Since the $3 \mathrm{D}$ and $\langle 3 \mathrm{D}\rangle$ models have, by construction, the same mean temperature structure, this allows the effects due to the horizontal temperature fluctuations to be singled out.

\section{Data}

As observational data we used two high-resolution, high signalto-noise ratio spectra of disk-centre solar intensity: that of Neckel \& Labs (1984) (hereafter referred to as the "Neckel intensity spectrum") and that of Delbouille et al. (1981) (hereafter referred to as the "Delbouille intensity spectrum" 5 ). We also used the solar flux spectrum of Neckel \& Labs (1984) and the solar flux spectrum of Kurucz $(2005 b)^{6}$, referred to as the "Kurucz flux spectrum".

\section{Data analysis}

We measured the equivalent width $(E W)$ of the lines using the IRAF task splot. We then computed the phosphorus abundance for the solar photosphere from the curve of growth of the line in question calculated with Linfor3D. To confirm our results, we fitted all observed line profiles with synthetic profiles. We found good agreement compared to the abundances derived from

\footnotetext{
3 http://wwwuser.oats.inaf.it/castelli/ sun/ap00t5777g44377k1asp.dat

4 http://www.aip.de/ mst/Linfor3D/

linfor_3D_manual.pdf

5 http://bass2000.obspm.fr/solaru_spect.php

${ }^{6}$ http://kurucz.harvard.edu/sun.html
} 


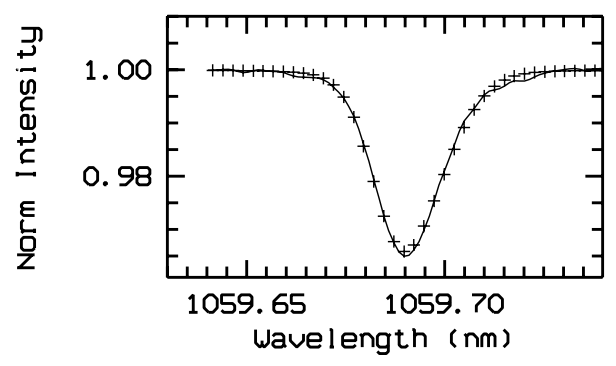

Fig. 1. The observed disk-centre, Neckel intensity spectrum (solid line) is plotted over the fit (crosses) obtained using a grid of synthetic spectra based on the $\mathrm{CO}^{5}$ BOLD model computed with Linfor3D.

the $E W \mathrm{~s}$. The remaining differences we found probably come from the difference between the continuum opacities used by SYNTHE and those used by Linfor3D.

The line profile fitting was done using a code described in Caffau et al. (2005), which performs a $\chi^{2}$ minimisation of the deviation between synthetic profiles and the observed spectrum. Figure 1 shows the fit of the solar intensity spectrum obtained from a grid of synthetic spectra synthesised with Linfor3D and based on the 3D $\mathrm{CO}^{5} \mathrm{BOLD}$ model. The close agreement between observed and synthetic line profiles indicates that the nonthermal (turbulent) line broadening is represented very well by the hydrodynamical velocity field of the $\mathrm{CO}^{5} \mathrm{BOLD}$ simulation.

\section{Results and discussion}

\subsection{The solar $P$ abundance}

The different sets of $\log g f$ values for the selected P I lines are very close, so that the derived solar $A(\mathrm{P})$ is quite insensitive to this choice. The solar phosphorus abundance varies by $0.05 \mathrm{dex}$, from the higher value obtained with the data set of Biemont et al. (1994), on the one hand, to the lower values of Biemont \& Grevesse (1973), Berzinsh et al. (corrected value of 1997), or the one from Kurucz \& Peytremann (1975). A lower value for the solar phosphorus abundance $(0.09$ dex below the one obtained using the Biemont et al. 1994 oscillator strength) can be obtained using the non-corrected values from Berzinsh et al. (1997).

With the (corrected) $\log g f$ of Berzinsh et al. (1997), we obtain $A(\mathrm{P})=5.443 \pm 0.058$ for the flux spectra considering the whole sample of five lines, $A(\mathrm{P})=5.426 \pm 0.064$ for the intensity spectra at disk-centre. The line at $1068.1 \mathrm{~nm}$ yields an abundance that is $1.6 \sigma$ below the mean value, while the four other lines are within one $\sigma$. If we remove this line from the computation, the standard deviation drops by a factor of two and we obtain $A(\mathrm{P})=5.467 \pm 0.029,5.450 \pm 0.042$ for flux and intensity spectra, respectively. The differences in the $A(\mathrm{P})$ determination from intensity and flux spectra could be due to NLTE corrections, which usually are different in intensity and flux. Unfortunately, there are no NLTE computations available for these lines. Our recommended solar photospheric abundance is $A(\mathrm{P})=5.458 \pm 0.036$, very close to all previous $1 \mathrm{D}$ determinations, but 0.1 dex higher than the value found by Asplund et al. (2005) using a 3D model.

The result of Asplund et al. (2005) is difficult to interpret since, according to our model, the $3 \mathrm{D}$ corrections for the $\mathrm{PI}$ lines in the Sun are small. Asplund et al. (2005) do not provide detailed information on the lines used or on their adopted $E W \mathrm{~s}$.

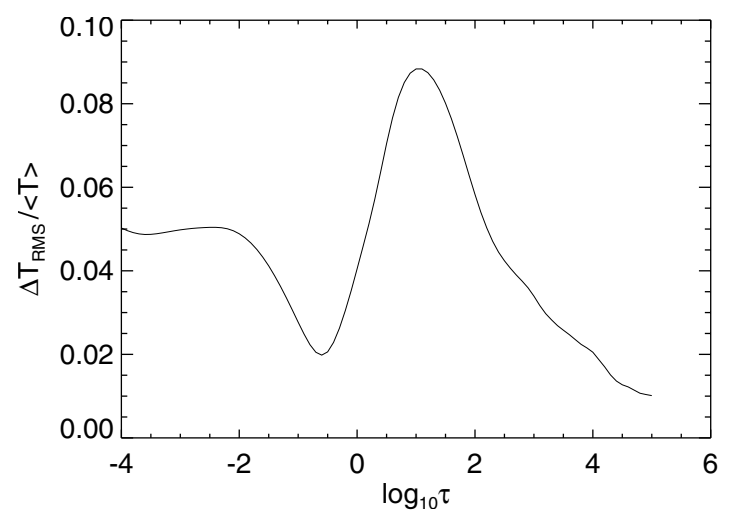

Fig. 2. Relative horizontal RMS temperature fluctuations (on surfaces of equal Rosseland optical depth) in the solar $\mathrm{CO}^{5} \mathrm{BOLD}$ model.

They adopt the $\log g f$ of Berzinsh et al. (1997), but they do not specify if they used the "corrected" or ab initio values. Had they used the latter values, the difference is easily understood; otherwise, we must conclude that the difference is due either to a difference between their $3 \mathrm{D}$ simulation and the $\mathrm{CO}^{5} \mathrm{BOLD}$ one or to a difference in the adopted $E W$ s. We point out here that our measured $E W$ s agre well with both those of Lambert \& Warner (1968), Lambert \& Luck (1978), and of Biemont et al. (1994)

A detailed summary of the solar phosphorus abundances we derived from individual lines, using different model atmospheres, is given in Table 4. Since the considered lines are not truly weak, the $3 \mathrm{D}$ corrections are unfortunately sensitive to the adopted micro-turbulence and, hence, must be interpreted with care. The 3D corrections only due to horizontal temperature fluctuations, indicated by the $3 \mathrm{D}-\langle 3 \mathrm{D}\rangle$ difference, are slightly positive throughout. In all lines, the $3 \mathrm{D}-\langle 3 \mathrm{D}\rangle$ difference for intensity spectra is systematically higher than for flux spectra, by roughly 0.02 dex. This is presumably due to the intensity spectra originating from somewhat deeper layers where horizontal fluctuations are larger. We note that the phosphorus lines are formed mostly in the range $-0.5<\log _{10} \tau_{\text {ross }}<0$, where the horizontal temperature fluctuations increase with increasing optical depth (see Fig. 2).

The 3D- $\langle 3 \mathrm{D}\rangle$ difference increases with the excitation potential of the line's lower level. In fact, the $3 \mathrm{D}-\langle 3 \mathrm{D}\rangle$ correction is rather small, not exceeding +0.030 dex for $\xi=1.0 \mathrm{~km} \mathrm{~s}^{-1}$ and +0.045 dex for $\xi=1.5 \mathrm{~km} \mathrm{~s}^{-1}$, which we consider as an upper limit.

The $3 \mathrm{D}-1 \mathrm{D}_{\mathrm{LHD}}$ corrections are systematically larger than the $3 \mathrm{D}-\langle 3 \mathrm{D}\rangle$ difference (by +0.01 to $0.02 \mathrm{dex}$ ), but within the same order of magnitude. From that we can conclude that the solar $\langle 3 \mathrm{D}\rangle$ and $1 \mathrm{D}_{\mathrm{LHD}}$ temperature profiles are very similar.

\section{2. $3 D$ effects on $P$ for other stars}

As shown above, we obtain only mild 3D effects in our solar phosphorus abundance analysis. They are comparable to the standard deviation (as listed in Col. (9) of Table 4) for the weak lines and a factor four larger than the standard deviation for the strongest line, so the 3D effects are hardly relevant. However, $3 \mathrm{D}$ effects should become stronger for metal-poor stars where the temperature profiles differ more strongly between $\langle 3 \mathrm{D}\rangle$ and $1 D_{\text {LHD }}$ models than for solar metallicity models (Asplund 2005). Very little work has been dedicated to 3D effects on the phosphorus abundance and none for metal-poor stellar models. To check 
the behaviour of the 3D abundance corrections, we investigated theoretically the phosphorus $3 \mathrm{D}-1 \mathrm{D}_{\mathrm{LHD}}$ relation in the resulting synthetic flux spectra for a few available $3 \mathrm{D} \mathrm{CO}^{5} \mathrm{BOLD}$ models.

We find, for three metal-poor models (6300/4.5/-2.0 $0^{7}$; $5900 / 4.5 /-3.0 ; 6500 / 4.5 /-3.0)$, that the difference in the mean temperature profile is the only factor contributing to the $3 \mathrm{D}$ correction. In the metal-poor models, just as in the solar model, the maximum contribution to the equivalent widths of the PI lines comes from a well-defined region close to the continuumforming layers. Even though the optical depth range that is contributing significantly is somewhat broader in the metal-poor models than in the solar model, the $\langle 3 \mathrm{D}\rangle$ and $1 \mathrm{D}_{\mathrm{LHD}}$ models are usually rather close in temperature in this region, so the effects of the different mean temperature structures are not very pronounced for the considered PI lines. The 3D correction related to the difference in the mean temperature structure of the $3 \mathrm{D}$ and $1 \mathrm{D}_{\text {LHD }}$ models amounts to $\approx+0.05$ dex for the -2.0 metallicity model and to $\approx+0.1$ dex for the most metal-poor model.

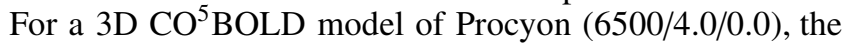
difference in the temperature profile of the $\langle 3 \mathrm{D}\rangle$ and $1 \mathrm{D}_{\mathrm{LHD}}$ model contributes to more than half of the total 3D correction. In Procyon, P I lines are formed at shallower optical depths than in the Sun by about $\Delta \log \tau \approx 0.3$. In this range the $1 \mathrm{D}_{\mathrm{LHD}}$ model is cooler than the $3 \mathrm{D} \mathrm{CO}^{5} \mathrm{BOLD}$ one. The related abundance correction depends on the excitation potential (strength) of the line, and it is still less than +0.030 dex for the strongest line. The contribution to the $3 \mathrm{D}$ correction related to the horizontal temperature fluctuations ranges from +0.022 to $+0.045 \mathrm{dex}$, increasing with the excitation potential (strength) of the line. In the range where the line is formed, the horizontal RMS temperature fluctuations in Procyon are of the order of 5\%, to be compared to $1 \%$ in the metal-poor models. The total $3 \mathrm{D}$ correction is thus less than +0.07 dex for all lines.

\section{Conclusions}

Using the $\mathrm{CO}^{5} \mathrm{BOLD}$ solar granulation model, we have determined the solar 3D LTE phosphorus abundance to be $A(\mathrm{P})=$ $5.46 \pm 0.04$, which compares very well to all previous determinations obtained using 1D models. This can be explained by the P I lines of Mult. 1 appearing to be rather insensitive to granulation effects, at least in the parameter interval explored by us ( $\mathrm{F}$ and $\mathrm{G}$ dwarfs of metallicity from solar to -3.0$)$. These lines can thus be useful for investigating the chemical evolution of phosphorus in the Galactic disk and in moderately metal-poor environments. Below $[\mathrm{P} / \mathrm{H}]=-1.0$, however, the lines have $E W \mathrm{~s}$ smaller than $0.5 \mathrm{pm}$, thus becoming exceedingly difficult to observe.

For the PI lines studied in this work, we find small (Sun) to moderate (Procyon, metal-poor stars) 3D abundance corrections. The sign of the corrections is found to be positive in all cases, meaning that the abundances resulting from a 3D analysis are larger than those obtained from a 1D model. Note that this behaviour is consistent with the results obtained by Steffen \& Holweger (2002) for S I, which has almost the same ionisation potential as PI (see their Fig. 8). In general, however, the magnitude and sign of the $3 \mathrm{D}$ effects depend on the properties of the absorbing ion (ionisation potential, excitation potential of the lower level, line strength, and wavelength) and on the thermodynamic structure of the stellar atmosphere. The results obtained in this work for PI must therefore not be generalised to other elements and other types of stars.
Acknowledgements. The authors L.S., H.-G.L., P.B. acknowledge financial support from EU contract MEXT-CT-2004-014265 (CIFIST). We acknowledge use of the supercomputing centre CINECA, which has granted us time to compute part of the hydrodynamical models used in this investigation, through the INAF-CINECA agreement 2006,2007.

\section{References}

Aller, L. H. 1949, ApJ, 109, 244

Anders, E., \& Grevesse, N. 1989, Geochim. Cosmochim. Acta, 53, 197 Andrievsky, S. M., Spite, M., Korotin, S. A., et al. 2007, A\&A, 464, 1081 Arnett, W. D. 1971, ApJ, 166, 153

Asplund, M. 2005, ARA\&A, 43, 481

Asplund, M., Grevesse, N., \& Sauval, A. J. 2005, Cosmic Abundances as Records of Stellar Evolution and Nucleosynthesis, ASP Conf. Ser., 336, 25

Baschek, B., \& Sargent, A. I. 1976, A\&A, 53, 47

Baschek, B., Scholz, M., Kudritzki, R. P., \& Simon, K. P. 1982, A\&A, 108, 387 Behr, B. B., Cohen, J. G., McCarthy, J. K., \& Djorgovski, S. G. 1999, ApJ, 517, L135

Berzinsh, U., Svanberg, S., \& Biemont, E. 1997, A\&A, 326, 412

Biemont, E., \& Grevesse, N. 1973, At. Data Nucl. Data Tables, 12, 217

Biemont, E., Martin, F., Quinet, P., \& Zeippen, C. J. 1994, A\&A, 283, 339

Bonifacio, P., Castelli, F., \& Hack, M. 1995, A\&AS, 110, 441

Bouret, J.-C., Lanz, T., \& Hillier, D. J. 2005, A\&A, 438, 301

Caffau, E., \& Ludwig, H.-G. 2007, A\&A, 467, L11

Caffau, E., Bonifacio, P., Faraggiana, R., et al. 2005, A\&A, 441, 533

Caffau, E., Faraggiana, R., Bonifacio, P., Ludwig, H.-G., \& Steffen, M. 2007, A\&A, 470, 699

Castelli, F., Parthasarathy, M., \& Hack, M. 1997, A\&A, 321, 254

Chayer, P., Vennes, S., Dupuis, J., \& Kruk, J. W. 2005, ApJ, 630, L169

Crowther, P. A., Hillier, D. J., Evans, C. J., et al. 2002, ApJ, 579, 774

Dobbie, P. D., Barstow, M. A., Hubeny, I., et al. 2005, MNRAS, 363, 763

Fremat, Y., \& Houziaux, L. 1997, A\&A, 320, 580

Freytag, B., Steffen, M., \& Dorch, B. 2002, Astron. Nachr., 323, 213

Garcia, Z. L., \& Levato, H. 1986, Astrophys. Lett., 25, 1

Grevesse, N., \& Sauval, A. J. 1998, Space Sci. Rev., 85, 161

Holweger, H. 1967, Z. Astrophys., 65, 365

Holweger, H., \& Mueller, E. A. 1974, Sol. Phys., 39, 19

Jahn, D., Rauch, T., Reiff, E., et al. 2007, A\&A, 462, 281

Kato, K.-I., Watanabe, Y., \& Sadakane, K. 1996, PASJ, 48, 601

Kaufmann, J. P., \& Schonberger, D. 1977, A\&A, 57, 169

Kurucz, R. 1993b, SYNTHE Spectrum Synthesis Programs and Line Data. Kurucz CD-ROM No. 18 (Cambridge, Mass.: Smithsonian Astrophysical Observatory), 18

Kurucz, R. L. 2005a, MSAIS, 8, 14

Kurucz, R. L. 2005b, MSAIS, 8, 189

Kurucz, R. L., \& Peytremann, E. 1975, SAO Special Rep., 362

Lambert, D. L., \& Warner, B. 1968, MNRAS, 138, 181

Lambert, D. L., \& Luck, R. E. 1978, MNRAS, 183, 79

Marcolino, W. L. F., Hillier, D. J., de Araujo, F. X., \& Pereira, C. B. 2007, ApJ, 654,1068

Mihalas, D. 1978, Stellar atmospheres, 2nd Edition (San Francisco: W. H. Freeman and Co), 650

Neckel, H., \& Labs, D. 1984, Sol. Phys., 90, 205

Nordlund, A., \& Stein, R. 1997, SCORe'96: Solar Convection and Oscillations and their Relationship, 225, 79

Ohl, R. G., Chayer, P., \& Moos, H. W. 2000, ApJ, 538, L95

Russell, H. N. 1929, ApJ, 70, 11

Sbordone, L. 2005, MSAIS, 8, 61

Sbordone, L., Bonifacio, P., Castelli, F., \& Kurucz, R. L. 2004, MSAIS, 5, 93

Seligman, C. E., \& Aller, L. H. 1970, Ap\&SS, 9, 461

Sion, E. M., Cheng, F. H., Sparks, W. M., et al. 1997, ApJ, 480, L17

Steffen, M., \& Holweger, H. 2002, A\&A, 387, 258

Takada-Hidai, M. 1991, Evolution of Stars: the Photospheric Abundance Connection, IAU Symp., 145, 137

Tobin, W., \& Kaufmann, J. P. 1984, MNRAS, 207, 369

Vennes, S., Chayer, P., Hurwitz, M., \& Bowyer, S. 1996, ApJ, 468, 898

Wedemeyer, S., Freytag, B., Steffen, M., Ludwig, H.-G., \& Holweger, H. 2004, A\&A, 414, 1121

Woosley, S. E., \& Weaver, T. A. 1995, ApJS, 101, 181

\footnotetext{
${ }^{7}$ Indicating $T_{\text {eff }} / \log g /[\mathrm{M} / \mathrm{H}]$.
} 
E. Caffau et al.: Solar phosphorus abundance, Online Material p 1

\section{Online Material}


E. Caffau et al.: Solar phosphorus abundance, Online Material p 2

Table 2. Spectroscopic determinations of the solar photospheric phosphorus abundance.

\begin{tabular}{lcl}
\hline \hline$A(\mathrm{P})$ & $\sigma$ & Reference \\
\hline 5.43 & & Lambert \& Warner (1968) \\
5.45 & 0.03 & Lambert \& Luck (1978) \\
5.45 & 0.04 & Anders \& Grevesse (1989) \\
5.45 & 0.06 & Biemont et al. (1994) \\
5.49 & 0.04 & Berzinsh et al. (1997) \\
5.45 & 0.04 & Grevesse \& Sauval (1998) \\
5.36 & 0.04 & Asplund et al. (2005) \\
5.46 & 0.04 & this work \\
\hline
\end{tabular}


E. Caffau et al.: Solar phosphorus abundance, Online Material p 3

Table 3. Oscillator strength for the IR phosphorus lines (wavelengths in $\mathrm{nm}$ ) available in the literature.

\begin{tabular}{rrrrrl}
\hline \hline & & $\log g f$ & & & Reference \\
1051.2 & 1053.0 & 1058.2 & 1059.7 & 1068.1 & \\
\hline-0.21 & 0.20 & 0.48 & -0.21 & -0.10 & Kurucz \& Peytremann (1975) \\
-0.23 & 0.18 & & -0.24 & -0.12 & NIST \\
-0.06 & 0.31 & 0.52 & -0.14 & -0.12 & Berzinsh et al. (1997) \\
-0.13 & 0.24 & 0.45 & -0.21 & -0.19 & Berzinsh et al. (1997) corr. \\
-0.10 & 0.27 & 0.49 & -0.17 & -0.15 & Biemont et al. (1994) \\
-0.24 & 0.17 & 0.45 & -0.24 & -0.13 & Biemont \& Grevesse (1973) \\
\hline
\end{tabular}

(NIST: http://physics.nist.gov/PhysRefData/ADS).

Table 4. Solar phosphorus abundances from the various observed spectra, adopting the (corrected) $\log g f$ of Berzinsh et al. (1997).

\begin{tabular}{|c|c|c|c|c|c|c|c|c|c|c|c|c|}
\hline \multirow{3}{*}{$\begin{array}{r}\text { Spectrum } \\
\text { (1) }\end{array}$} & \multirow{3}{*}{$\begin{array}{r}\text { Wave } \\
{[\mathrm{nm}]} \\
(2)\end{array}$} & \multirow{3}{*}{$\begin{array}{r}E W \\
{[\mathrm{pm}]} \\
\\
(3)\end{array}$} & \multirow{3}{*}{$\begin{array}{r}{[\mathrm{dex}]} \\
3 \mathrm{D} \\
(4)\end{array}$} & \multicolumn{3}{|c|}{$A(\mathrm{P})$ from $E W$} & \multirow[b]{2}{*}{ [dex] } & \multirow{3}{*}{$\begin{array}{r}\sigma \\
{[\operatorname{dex}]} \\
\\
\\
(9)\end{array}$} & \multicolumn{2}{|c|}{$3 \mathrm{D}-1 \mathrm{D}_{\mathrm{LHD}}$} & \multicolumn{2}{|c|}{$3 \mathrm{D}-\langle 3 \mathrm{D}\rangle$} \\
\hline & & & & $\begin{array}{r}{[\mathrm{dex}]} \\
\mathrm{AT}]\end{array}$ & $\begin{array}{l}{[\mathrm{dex}]} \\
\mathrm{AS}\end{array}$ & $\begin{array}{r}{[\operatorname{dex}]} \\
\mathrm{H}\end{array}$ & & & {$[\mathrm{dex}]$} & [dex] & {$[\operatorname{dex}]$} & [dex] \\
\hline & & & & $\begin{array}{l}1.0 \\
(5)\end{array}$ & $\begin{array}{l}1.5 \\
(6)\end{array}$ & $\begin{array}{l}1.0 \\
(7)\end{array}$ & $\begin{array}{l}1.5 \\
(8)\end{array}$ & & $\begin{array}{r}1.0 \\
(10)\end{array}$ & $\begin{array}{r}1.5 \\
(11)\end{array}$ & $\begin{array}{r}1.0 \\
(12)\end{array}$ & $\begin{array}{r}1.5 \\
(13)\end{array}$ \\
\hline $\mathrm{KF}$ & 1051.2 & 0.674 & 5.482 & 5.476 & 5.471 & 5.480 & 47 & 0.015 & 0.015 & 0.019 & 0.005 & 0.010 \\
\hline NF & 1051.2 & 0.734 & 5.523 & 5.517 & 5.512 & 5.521 & 5.516 & 0.013 & 0.015 & 0.020 & 0.005 & 0.010 \\
\hline NI & 1051.2 & 0.811 & 5.502 & 5.476 & 5.473 & 5.498 & 5.493 & 0.012 & 0.028 & 0.032 & 0.022 & 0.026 \\
\hline DI & 1051.2 & 0.822 & 5.508 & 5.483 & 5.479 & 5.504 & 5.500 & 0.012 & 0.028 & 0.032 & 0.022 & 0.027 \\
\hline $\mathrm{KF}$ & 1053.0 & 1.330 & 5.431 & 421 & 5.412 & 5.427 & 5.417 & 0.010 & 0.019 & 00 & 0.005 & 0.015 \\
\hline NF & & 1.340 & & 5.424 & 5.416 & 5.431 & 5.421 & 0.010 & 0.019 & 28 & 0.005 & 0.015 \\
\hline NI & 1053.0 & 1.520 & 5.425 & 5.392 & 5.382 & 5.417 & 5.408 & 0.010 & 0.036 & 0.044 & 0.025 & 0.034 \\
\hline DI & 1053.0 & 1.550 & 5.436 & 5.402 & 5.392 & 5.427 & 5.418 & 0.010 & 0.036 & 0.044 & 0.025 & 0.034 \\
\hline $\mathrm{KF}$ & 58.2 & 2.280 & 5.480 & 5.463 & 5.448 & 5.473 & 5.456 & 0.012 & 0.027 & 00 & 0.006 & 0.023 \\
\hline NF & 1058.2 & 2.120 & 5.436 & 5.421 & 5.406 & 5.429 & 5.414 & 0.013 & 0.025 & 0.039 & 0.005 & 0.021 \\
\hline NI & 1058.2 & 2.330 & 5.407 & 5.363 & 5.346 & 5.393 & 5.379 & 0.012 & 0.047 & 0.059 & 0.029 & 0.043 \\
\hline DI & 1058.2 & 2.330 & 5.407 & 5.363 & 5.346 & 5.393 & 5.379 & 0.012 & 0.047 & 0.059 & 0.029 & 0.043 \\
\hline $\mathrm{KF}$ & 9.7 & 0.684 & 82 & 175 & 5.471 & 5.480 & 5.47 & 014 & 0.015 & 0 & 0.005 & 0.010 \\
\hline NF & 9.7 & 0.660 & 5.464 & 5.458 & 5.454 & 5.462 & 5 & 0.014 & 0.015 & 0. & 0.005 & 0.010 \\
\hline NI & 1059.7 & 0.748 & 5.456 & 5.431 & 5.427 & 5.452 & 5.448 & 0.012 & 0.027 & 0.030 & 0.022 & 0.026 \\
\hline DI & 1059.7 & 0.751 & 5.458 & 5.433 & 5.429 & 5.454 & 5.450 & 0.012 & 0.027 & 0.031 & 0.022 & 0.026 \\
\hline KF & 8.1 & 0.670 & & 64 & 5.360 & 5.368 & & 0.014 & 0.015 & & 0.005 & 0.010 \\
\hline NF & 8.1 & 0.617 & & 5.324 & 5.320 & 5.328 & 5.324 & & 0.015 & & 0.006 & 0.010 \\
\hline NI & 1068.1 & 0.699 & 5.322 & 5.297 & 5.294 & 5.318 & 5.314 & 0.013 & 0.027 & 0.030 & 0.022 & 0.026 \\
\hline DI & 1068.1 & 0.731 & 5.343 & 5.319 & 5.315 & 5.340 & 5.336 & 0.013 & 0.027 & 0.030 & 0.022 & 0.026 \\
\hline
\end{tabular}

Column (1) spectrum identification: DI: Delbouille intensity, NI: Neckel intensity, NF: Neckel flux, KF: Kurucz flux. Column (2) wavelength of the line. Column (3) measured equivalent width. Column (4) phosphorus abundance, $A(\mathrm{P})$, according to the $\mathrm{CO}^{5} \mathrm{BOLD} 3 \mathrm{D}$ model. Columns (5)-(8) provide $A(\mathrm{P})$ from 1D models, odd numbered columns correspond to a micro-turbulence $\xi$ of $1.0 \mathrm{~km} \mathrm{~s}^{-1}$, even numbered columns to $\xi=1.5 \mathrm{~km} \mathrm{~s}{ }^{-1}$. Column (9) uncertainty in $A(\mathrm{P})$ due to the uncertainty in the measured $E W$ s. Column (10)-(13) provide 3D corrections, even numbered columns for $\xi=1.0 \mathrm{~km} \mathrm{~s}^{-1}$, and odd numbered columns for $1.5 \mathrm{~km} \mathrm{~s}^{-1}$, respectively. 